\title{
ARTICLE
}

\section{Magnetization study on the ising ferromagnet URhGe with high-precision angle-resolved magnetic field near the hard axis}

\author{
Shota Nakamura $^{\mathrm{a}^{*}}$, Toshiro Sakakibara ${ }^{\mathrm{a}}$, Yusei Shimizu ${ }^{\mathrm{a}, \mathrm{b}}$, Shunichiro Kittaka ${ }^{\mathrm{a}}$, Yohei Kono ${ }^{\mathrm{a}}$, \\ Yoshinori Haga $^{c}$, Jiří Pospíšil ${ }^{\mathrm{c}, \mathrm{d}}$ and Etsuji Yamamoto ${ }^{\mathrm{c}}$ \\ ${ }^{a}$ Institute for Solid State Physics, The University of Tokyo, Kashiwa 277-8581, Japan; ${ }^{b}$ Institute for Materials Research, Tohoku \\ University, Oarai 311-1313, Japan; ' Japan Atomic Energy Agency, Tokai 319-1106, Japan; ${ }^{d}$ Charles University in Prague, \\ Faculty of Mathematics and Physics, DCMP, Ke Karlovu 5, 12116 Prague 2, Czech Republic
}

\begin{abstract}
Dc magnetization measurements have been performed on the orthorhombic Ising ferromagnet URhGe with high-precision control of the magnetic field direction near the hard $b$ axis. The ferromagnetic moment of this compound, which aligns along the $c$ axis in zero field, is known to exhibit a reorientation transition in the $b$-axis magnetic field $H_{b}$. We observed a clear first-order reorientation transition with a small hysteresis, and estimated that the tricritical point TCP is located above $4 \mathrm{~K}$ at least, at $\mu_{0} H_{\mathrm{b}} \sim 10.5 \mathrm{~T}$ and the wing quantum critical points exist at $\mu_{0}\left(H_{b}, H_{c}\right) \sim(13.5 \mathrm{~T}, \pm 1 \mathrm{~T})$. We have also measured the longitudinal magnetostriction and thermal expansion of URhGe with $H$ along the $b$ axis, and observed that the lattice shrinks along the $b$ axis upon the transition. The magnetization and the thermal expansion data indicate the presence of a characteristic temperature $\sim 1 \mathrm{~K}$.
\end{abstract}

Keywords: quantum phase transition; ferromagnetic superconductor; pairing mechanism; magnetization measurement; metamagnetism

\section{Introduction}

Recently, a ferromagnetic (FM) quantum phase transition (QPT) in clean metals has attracted much interest because a first-order QPT is commonly observed at low temperatures below a tricritical point (TCP) [1] Below the TCP temperature $T_{\mathrm{TCP}}, \mathrm{FM}$ first-order planes ("wings") appear in the three-dimensional phase diagram [2]. This type of " $T-p-H$ phase diagram" with pressure $p$ as a tuning parameter has been studied in itinerant FM compounds, such as $\mathrm{UGe}_{2}$, URhAl, UCoGa, and $\mathrm{ZrZn}_{2}$ [3-6]. Whereas much theoretical work has been conducted, experiments on the wing structure phase diagram have so far been performed to much less extent because a high pressure is normally required to access the FM QPT. By contrast, as a magnetic field perpendicular to the magnetic easy axis being the tuning parameter, URhGe provides a good opportunity to investigate the whole FM phase diagram, in particular the wing structure $[7,8]$.

We focus in this paper on the magnetic properties of the itinerant Ising ferromagnet URhGe, which has the orthorhombic TiNiSi type crystal structure with the space group Pnma. The magnetic moment $M$ of $0.4 \mu_{\mathrm{B}} / \mathrm{U}$ aligns along the $c$ axis, the magnetization easy axis, below the Curie temperature $T_{\mathrm{C}}$ of $9.5 \mathrm{~K}[9,10]$. The $T_{\mathrm{C}}$ of this compound can be tuned to zero by applying a magnetic field along the $b$ axis, perpendicular to the spontaneous moment. Under the $b$-axis field $H_{b}$, the "ferromagnetic transition" is such that the $c$-axis component of the moment appears below $T_{\mathrm{C}}\left(H_{b}\right)$. This spin reorientation transition becomes first order below $T_{\mathrm{TCP}}$, and $T_{\mathrm{C}}\left(H_{b}\right)$ decreases to zero at the critical field $H_{\mathrm{R}}(T=0) \sim 12 \mathrm{~T}[9,10]$. On cooling below $T_{\mathrm{TCP}}$, the second-order transition line bifurcates into two by an application of a $c$-axis field $H_{c}$, and a wing structure appears in the $T-H_{c^{-}} H_{b}$ phase diagram [8]. Very interestingly, re-entrant superconductivity (RSC) emerges around the "wings" below the critical temperature $T_{\mathrm{RSC}} \sim 0.42 \mathrm{~K}$ [7]. The origin of RSC has been discussed in the light of FM fluctuations near the spin reorientation transition [8] with an assumption of very low $T_{\mathrm{TCP}}[7]$. Experimentally, however, the location of TCP in URhGe has been controversial $[11,12]$. In the present paper, we have performed low temperature dc magnetization and thermal expansion measurements on URhGe under a precise control of the field angle to investigate the wing structure, in particular the location of the TCP in the three-dimensional $T-H_{c^{-}} H_{b}$ phase diagram.

*Corresponding author. Email: sna@issp.u-tokyo.ac.jp 

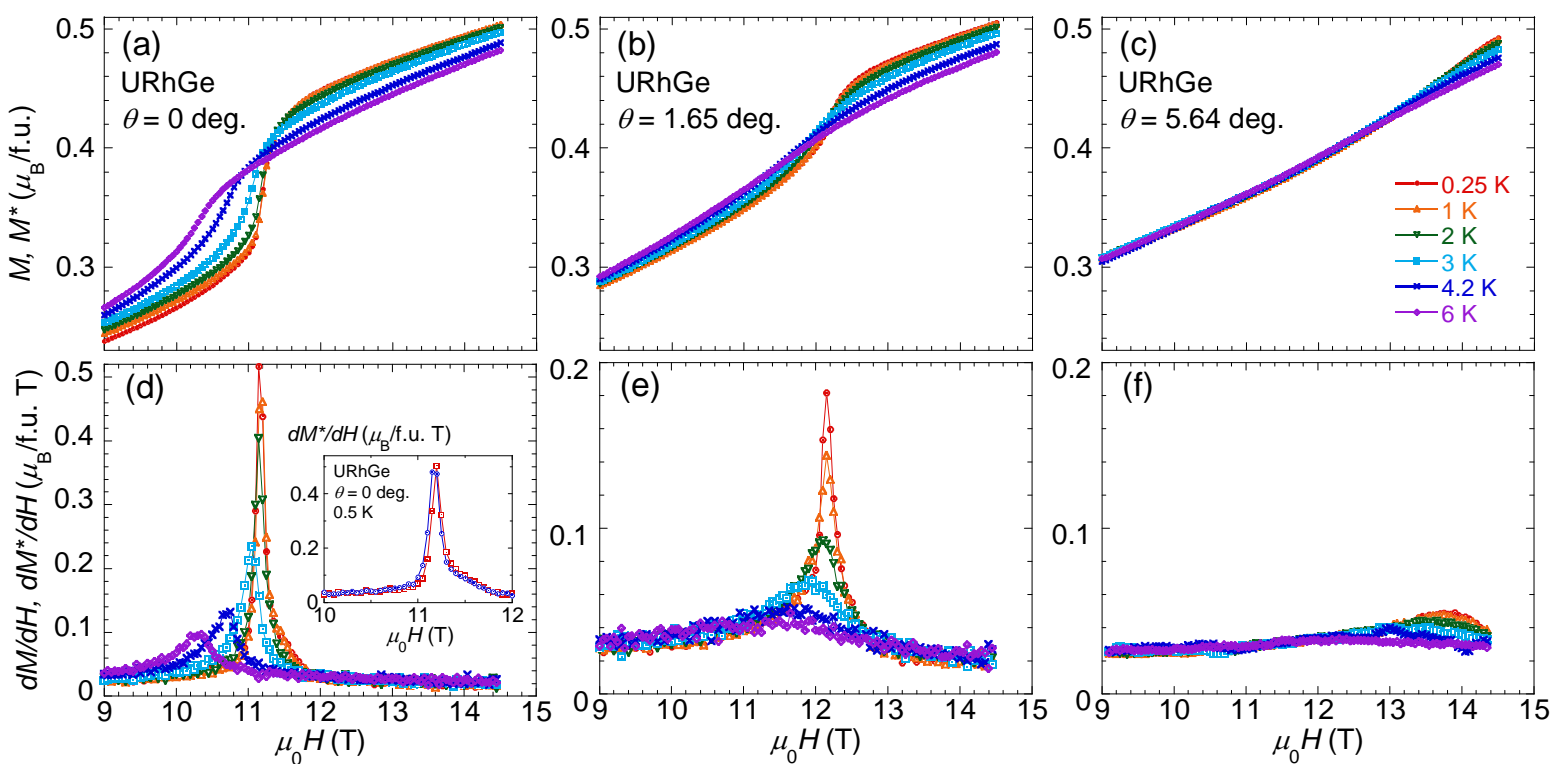

Figure 1. Magnetization curves $M(H)$ of URhGe near $H_{\mathrm{R}}(\theta)$ at $0.25,1,2,3,4.2$, and $6 \mathrm{~K}$, measured at an angle $\theta$ of (b) $1.65^{\circ}$ and (c) $5.64^{\circ}$, together with their differential curves $d M / d H$ for (e) $1.65^{\circ}$ and (f) $5.64^{\circ}$, where $\theta$ is measured from the $b$ towards the $c$ axes. For comparison, $M^{*}$ and $d M^{*} / d H$ at $\theta=0^{\circ}$, which contains some torque contributions, are also plotted in Figures. $1(\mathrm{a})$ and (d). The inset in Figure 1 (d) shows $d M^{*} / d H$ at $0.5 \mathrm{~K}$ and $\theta=0^{\circ}$ for both up- and down-field sweeps, indicating the presence of a small hysteresis.

\section{Experimental procedures}

Single-crystalline sample of URhGe was grown by Czochralski method and cut into a rectangular shape with dimentions $a \times b \times c=0.7 \times 1.0 \times 0.5 \mathrm{~mm}^{3}$ with 4.4 mg mass at JAEA. The present sample does not exhibit superconductivity. Whereas superconductivity can be observed only in stoichiometric samples of URhGe with a very small residual resistivity [13], the FM transition is known to be much more robust and does not change even in doped systems, $\mathrm{URh}_{0.9} \mathrm{Co}_{0.1} \mathrm{Ge}$ and $\mathrm{URh}_{\mathrm{x}} \mathrm{Ir}_{1-\mathrm{x}} \mathrm{Ge}$ $[8,14]$.

Dc magnetization measurements were performed by means of capacitively-detected Faraday magnetometer [15]. In this method, we detect a magnetic force $M d H_{z} / d z$ that the sample experiences in a field gradient $d H_{z} / d z$, where the $z$ axis denotes the vertical axis along which magnetic fields are generated by superconducting solenoid. Normally, a magnetic torque contribution appears in the output of the capacitance transducer, and is subtracted by measuring the torque background by setting $d H_{z} / d z=0$. The details of this method can be found in Ref. [15]. The magnetrostriction measurements have been performed by using capasitive dilatometer.

We have developed a two-axis rotation device, consisting of a piezo-stepper-driven goniometer combined with a home-made tilting stage [16]. Installation of the device into the magnetometer has achieved an in-situ alignment of the sample within an accuracy of $0.1 \mathrm{deg}$. The details of the rotation device will be published elsewhere. In this paper, we measured $\theta$ dependence of the magnetization in the $b c$ plane in the range $|\theta|<6^{\circ}$, where $\theta$ is the angle of the magnetic field measured from the $b$ axis. A ${ }^{3} \mathrm{He}-{ }^{4} \mathrm{He}$ dilution refrigerator is used for cooling the sample.

\section{Results}

\subsection{Magnetization}

Figure 1 shows the magnetization curves $M(H)$ of URhGe near $H_{\mathrm{R}}(\theta)$ at $0.25,1,2,3,4.2$, and $6 \mathrm{~K}$, measured at $\theta$ of (b) $1.65^{\circ}$ and (c) $5.64^{\circ}$, together with their differential curves $d M / d H$ for (e) $1.65^{\circ}$ and (f) 5.64 $4^{\circ}$ The corresponding data for $\theta=0^{\circ}, M^{*}$ and $d M^{*} / d H$, are also plotted in Figures 1 (a) and 1(d), respectively. Here we denote the magnetization at $\theta=0^{\circ}$ by $M^{*}(H)$ to distinguish from that for $\theta>0.1^{\circ}$, because some torque contribution remains in the magnetization data of the FM state below $H_{\mathrm{R}}$ at $|\theta|<0.1^{\circ}$ due to the following reason. Maxwell equation $(\operatorname{div} B=0)$ implies that $d H_{x} / d x=d H_{y} / d y=-(1 / 2) d H_{z} / d z$ holds at the magnet center. The vertical field gradient $G_{z}\left(=d H_{z} / d z\right)=8 \mathrm{~T} / \mathrm{m}$ then causes a field gradient of order $4 \mathrm{~T} / \mathrm{m}$ along the horizontal direction as well; when the sample with its vertical $b$ axis is slightly off-centered by $1 \mathrm{~mm}$ along the horizontal $c$ direction, it experiences a magnetic field of $4 \mathrm{mT}$ along the $c$ axis. This tiny additional field proportional to $G_{z}$ is not so disturbing near $H_{\mathrm{R}}$ for $|\theta|$ $0.1^{\circ}$, because $H_{\mathrm{c}}$ is already large enough for the ferromagnetic domains to align towards either $+c$ or $-C$ directions. However, for $|\theta| 0.1^{\circ}$, the sample is still in the FM domain state even near $H_{R}$, so that the domain population significantly changes by the small additional field. As a consequence, the torque contribution, which predominantly arises from the $c$-axis component of the magnetization, significantly changes with $G_{z}$. The torque background then becomes different between $G_{z}=8 \mathrm{~T} / \mathrm{m}$ and 0 , resulting in an incomplete torque subtraction. We expect that $d M^{*} / d H$ peak height in Figure 1 (d) is about $30 \%$ overestimated due to this effect. 


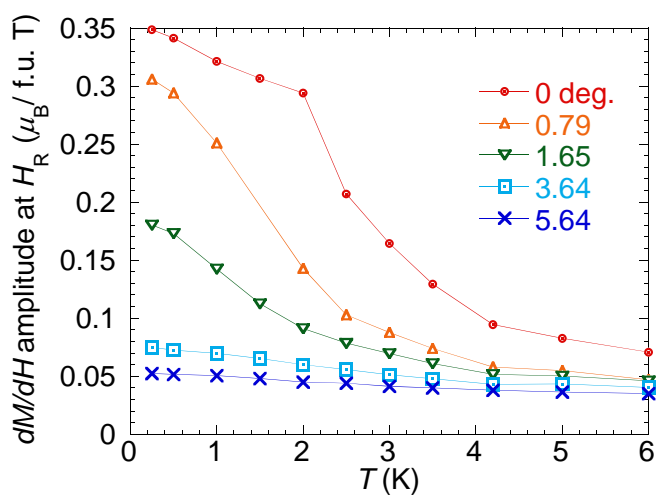

Figure 2. Temperature dependence of the peak amplitude of $d M / d H$ at $H_{\mathrm{R}}$ obtained at $\theta=0^{\circ}, 0.79^{\circ}, 1.65^{\circ}, 3.64^{\circ}$, and $5.64^{\circ}$. The data points at $\theta=0^{\circ}$ are reduced by a factor 0.7 to compare with those for $\theta \neq 0^{\circ}$, as explained in the text.

As can be seen from Figures 1(a) and 1(d) $\left(\theta=0^{\circ}\right.$ data), the spin reorientation transition occurs at $\mu_{0} H_{\mathrm{R}} \sim$ $11.2 \mathrm{~T}$ for $T=0.25 \mathrm{~K}$, and just above $H_{\mathrm{R}}$ the magnetization value reaches $\sim 0.46 \mu_{\mathrm{B}} / \mathrm{U}$, almost equal to the value of spontaneous moment along the $c$ axis in zero field. The inset in Figure 1(d) shows that a small hysteresis appears in the transition field at $0.5 \mathrm{~K}$ and $\theta=$ $0^{\circ}$. The presence of the hysteresis provides strong evidence for the first-order transition. We observed that the hysteresis becomes indiscernible above $1 \mathrm{~K}$. The $d M / d H$ peak height also becomes smaller and broader with increasing temperature, but the peak feature of $d M / d H$ remains even at $6 \mathrm{~K}$. With increasing $\theta$, the $d M / d H$ peak becomes smaller, and shifts to the higher field side. The peak feature disappears above $4.2 \mathrm{~K}$ for $\theta$ $=1.65^{\circ}$ and above $0.25 \mathrm{~K}$ for $\theta=5.64^{\circ}$, indicating that these points are located just outside the wing boundary.

Figure 2 shows the temperature dependence of the peak height of $d M / d H$ at $H_{\mathrm{R}}$ obtained at $\theta=0^{\circ}, 0.79^{\circ}$, $1.65^{\circ}, 3.64^{\circ}$, and $5.64^{\circ}$. The data points at $\theta=0^{\circ}$ are reduced in magnitude by a factor of 0.7 to correct the residual torque contribution as described above. A rapid decrease of the amplitude can be seen above $2 \mathrm{~K}$ for $\theta=$ $0^{\circ}$ and above $0.5 \mathrm{~K}$ for $\theta=0.79^{\circ}$ and $1.65^{\circ}$. For $\theta=$ $3.64^{\circ}$ and $5.64^{\circ}$, the amplitude is not sensitive to temperature. Further details of this magnetization results will be given in Ref. [17].

\subsection{Thermal expansion and magnetostriction}

Figure 3 shows the longitudinal magnetostriction of URhGe at $0.5 \mathrm{~K}$ and $2 \mathrm{~K}$ in magnetic fields along the $b$ axis. A first-order like transition is observed at $\mu_{0} H_{\mathrm{R}} \sim$ 12.5 $\mathrm{T}$ in Figure 3. Note that the two-axis rotation device was not used in the magnetostriction measurements. The higher value of $H_{\mathrm{R}}$ compared to that by the present magnetization measurements can be ascribed to a misalignment of the sample, which is roughly estimated to be $\sim 2^{\circ}$ towards the $c$ axis. The magnetostriction indicates that the lattice significantly shrinks along the $b$ axis upon the reorientation transition. The inset shows the thermal expansion relative to the value at $0.25 \mathrm{~K}$,

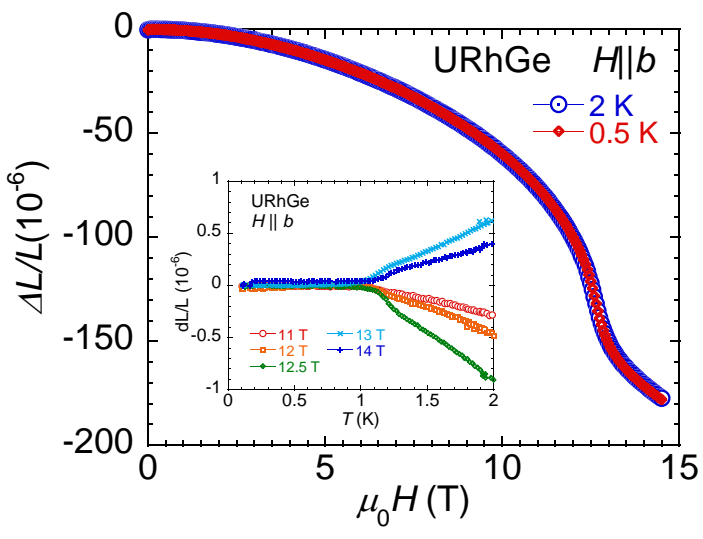

Figure 3. Longitudinal magnetostriction of URhGe at 0.5 $\mathrm{K}$ and $2 \mathrm{~K}$ in magnetic fields along the $b$ axis. Note that there is a misalignment of the magnetic field direction from the $b$ axis by $\sim 2^{\circ}$ towards the $c$ axis. The inset shows thermal expansion (relative to the value at $0.25 \mathrm{~K}$ ) at 11,12 , $12.5,13$, and $14 \mathrm{~T}$ with the same configuration as in the magnetostriction measurements.

measured at $11,12,12.5,13$, and $14 \mathrm{~T}$ with the same configuration as the magnetostriction measurements. We note that thermal expansion is smaller than the magnetostriction by two orders of magnitude. The thermal expansion is vanishingly small below $1 \mathrm{~K}$ in the measured field range, and spread with each other depending on the field values above $1 \mathrm{~K}$. These results indicate that the magnetostriction curves near the reorientation transition are almost temperature independent below $1 \mathrm{~K}$, and broadening of the reorientation transition occurs above $1 \mathrm{~K}$ as observed in the present magnetization results.

\section{Discussion}

At the base temperature of $0.25 \mathrm{~K}$, the peak amplitude of $d M / d H(\theta)$ at $H_{\mathrm{R}}(\theta)$ decreases progressively as $\theta$ increases, making it somewhat difficult to precisely determine the edge of the wings from the present data. Nevertheless, we estimate from Figure 2 that the wing edge extends to the range $3.64^{\circ}<\theta<5.64^{\circ}$, since beyond this range the peak feature of $d M / d H$ suddenly becomes very weak and is only weakly temperature dependent. From these results, we expect that the $T=0$ quantum wing critical points (QWCP) are located near $\mu_{0}\left(H_{b}, H_{c}\right) \sim(13.5 \mathrm{~T}, \pm 1 \mathrm{~T})$, i.e., $|\theta| \sim 5^{\circ}$.

From Figures 1 and 2, the $d M / d H$ amplitude becomes weaker near $3 \mathrm{~K}$ at $\theta=0^{\circ}$, and the linear extrapolation value of $d M / d H$ amplitude between 2 and $3 \mathrm{~K}$ reaches to 0.05 , which is the value of $\theta=5.64^{\circ}$, at $\sim 4 \mathrm{~K}$. We can estimate that the position of TCP is above $4 \mathrm{~K}$ at least. Note that a weak peak feature of $d M / d H$ is observed even between 4.2 and $6 \mathrm{~K}$ at $\theta=0^{\circ}$, indicating that TCP may locate above $6 \mathrm{~K}$. It turns out, then, that the ratio of $T_{\mathrm{TCP}} / T_{\mathrm{RSC}}$ is rather large in URhGe, $T_{\mathrm{TCP}} / T_{\mathrm{RSC}} \gtrsim 10$, suggesting that the effect of fluctuations around TCP on RSC may be weaker than expected in Ref. [18]. It is likely that the RSC is rather associated with its 
unusually weak first-order nature at the transition much below $T_{\mathrm{TCP}}$.

Despite the rather high $T_{\mathrm{TCP}}$, our magnetization and magnetostriction data suggest the presence of a characteristic temperature of $\sim 1 \mathrm{~K}$; temperature dependences of $M(H)$ as well as the magnetistriction become very weak below $T \sim 1 \mathrm{~K}$, as shown in Figures 2 and 3. A similar characteristic temperature has been reported in lower fields between 0 and $7 \mathrm{~T}$ in the previous thermoelectric power measurements [11] and has been assigned to be a crossover into a coherent low temperature Fermi-liquid regime

It should be stressed that the first-order reorientation transition in URhGe is very weak, as demonstrated by the very small hysteresis and a rapid weakening of the transition on approaching the wing edges. Owing to the weakness of the transition, substantial fluctuations of the order parameter might remain below the characteristic temperature of $1 \mathrm{~K}$.

\section{Conclusion}

We have examined the angle-resolved magnetization of the orthorhombic Ising ferromagnet URhGe near the $b$ axis under precise control of the magnetic field direction. We observed a clear first-order reorientation transition, and determined the profiles of the wing structure phase diagram, in particular the location of a tricritical point TCP and quantum wing critical points QWCPs. We estimate that TCP is located above $4 \mathrm{~K}$ at least, an order of magnitude higher than the critical temperature of reentrant superconductivity (RSC). This fact suggests that ferromagnetic fluctuations associated with TCP are not the main cause of RSC in this system. The present magnetization as well as the magnetostriction data indicate the presence of a rather low energy scale of $\sim 1 \mathrm{~K}$, which is almost the same with the temperature scale of a Fermi-liquid regime reported in the previous thermoelectric power measurements [11]. Interestingly, the first-order transition remains very weak in this temperature region. Such a weakness of the first-order transition might host substantial fluctuations even at low temperatures, leading to RSC in this system.

\section{Acknowledgments}

We thank N. Tang, Y. Qui, and S. Nakatsuji for technical support of X-ray structural analysis. The present work was supported in part by a Grant-in-Aid for Scientific Research on Innovative Areas "J-Physics" (15H05883) and KAKENHI (15H03682) from MEXT.

\section{References}

[1] M. Brando, D. Belitz, F.M. Grosche, T.R. Kirkpatrick, Metallic quantum ferromagnets, Rev. Mod. Phys. 88 (2016), p. 025006.

[2] R.B. Griffiths, Proposal for notation at tricritical points, Phys. Rev. B 7 (1973), pp. 545-551.

[3] C. Pfleiderer, A.D. Huxley, Pressure dependence of the magnetization in the ferromagnetic superconductor $\mathrm{UGe}_{2}$, Phys. Rev. Lett. 89 (2002) p. 147005.

[4] M. Uhlarz, C. Pfleiderer and S.M. Hayden, Quantum phase transitions in the itinerant ferromagnet $\mathrm{ZrZn}_{2}$, Phys. Rev. Lett. 93 (2004), p. 256404.

[5] Y. Shimizu, D. Braithwaite, B. Salce, T. Combier, D. Aoki, E.N. Hering, S.M. Ramos and J. Flouquet, Unusual strong spin-fluctuation effects around the critical pressure of the itinerant Ising-type ferromagnet URhAl, Phys.Rev. B 91 (2015), p. 125115.

[6] M. Míšek, J. Prokleška, P. Opletal, P. Proschek, J. Kaštil, J. Kamarád and V. Sechovský, Pressure-induced quantum phase transition in the itinerant ferromagnet UCoGa, AIP Advances 7 (2017), p. 055712.

[7] F. Lévy, I. Sheikin, B. Grenier and A.D. Huxley, Magnetic field-induced superconductivity in the ferromagnet URhGe, Science 309 (2005), pp. 1343-1346.

[8] Y. Tokunaga, D. Aoki, H. Mayaffre, S. Krämer, M.-H. Julien, C. Berthier, M. Horvatić, H. Sakai, S. Kambe and S. Araki, Reentrant superconductivity driven by quantum tricritical fluctuations in URhGe: Evidence from ${ }^{59} \mathrm{Co} \mathrm{NMR}$ in $\mathrm{URh}_{0.9} \mathrm{Co}_{0.1} \mathrm{Ge}$, Phys. Rev. Lett. 114 (2015), p. 216401.

[9] F. Hardy, D. Aoki, C. Meingast, P. Schweiss, P. Burger, H. v. Löhneysen and J. Flouquet, Transverse and longitudinal magnetic-field responses in the ising ferromagnets URhGe, UCoGe, and $\mathrm{UGe}_{2}$, Phys. Rev. B 83 (2011), p. 195107.

[10] D. Aoki and J. Flouquet, Superconductivity and ferromagnetic quantum criticality in uranium compounds, J. Phys. Soc. Jpn. 83 (2014), p. 061011.

[11] A. Gourgout, A. Pourret, G. Knebel, D. Aoki, G. Seyfarth and J. Flouquet, Collapse of ferromagnetism and fermi surface instability near reentrant superconductivity of URhGe, Phys. Rev. Lett. 117 (2016), p. 046401.

[12] H. Kotegawa, K. Fukumoto, T. Toyama, H. Tou, H. Harima, A. Harada, Y. Kitaoka, Y. Haga, E. Yamamoto, Y. Ōnuki, et al., ${ }^{73} \mathrm{Ge}$-nuclear magnetic resonance/nuclear quadrupole resonance investigation of magnetic properties of URhGe, $J$. Phys. Soc. Jpn. 84 (2015), p. 054710.

[13] A. Miyake, D. Aoki and J. Flouquet, Field re-entrant superconductivity induced by the enhancement of effective mass in URhGe, J. Phys. Soc. Jpn. 77 (2008), p. 094709.

[14] J. Pospíšil, Y. Haga, S. Kambe, Y. Tokunaga, N. Tateiwa, D. Aoki, F. Honda, A. Nakamura, Y. Homma, E. Yamamoto and T. Yamamura, Switching of magnetic ground states across the 
$\mathrm{UIr}_{1-\mathrm{x}} \mathrm{Rh}_{\mathrm{x}} \mathrm{Ge}$ alloy system, Phys. Rev. B 95 (2017), p. 155138.

[15] T. Sakakibara, H. Mitamura, T. Tayama and H. Amitsuka, Faraday force magnetometer for high-sensitivity magnetization measurements at very low temperatures and high fields, Jpn. J. Appl. Phys. 33 (1994), p. 5067.

[16] S. Kittaka, A. Kasahara, T. Sakakibara, D. Shibata, S. Yonezawa, Y. Maeno, K. Tenya and K. Machida, Sharp magnetization jump at the firstorder superconducting transition in $\mathrm{Sr}_{2} \mathrm{RuO}_{4}$, Phys. Rev.
B 90 (2014), p. 220502, Supplemental Material.

[17] S. Nakamura, T. Sakakibara, Y. Shimizu, S. Kittaka, Y. Kono, Y. Haga, J. Pospíšil and E. Yamamoto, Wing structure in the phase diagram of the Ising ferromagnet URhGe close to its tricritical point investigated by angle-resolved magnetization measurements, Phys. Rev. B. 96 (2017), p. 094411.

[18] V.P. Mineev, Reentrant superconductivity in URhGe, Phys. Rev. B. 91 (2015), p. 014506. 